\title{
THE CO-DESIGN COGNITIVE PROCESS: IMPACTS OF A SPATIAL AUGMENTED REALITY PLATFORM
}

\author{
M. Poulin ${ }^{凶}$, C. Masclet and J.-F. Boujut \\ Grenoble INP, France \\ $\square$ maud.poulin@grenoble-inp.fr
}

\section{Abstract}

The study of design cognitive activity began in the 70s under the influence of psychology and ergonomics. Since then, the design process has undergone many changes with the advent of technology. This paper will notably present you to one of them: the Spatial Augmented Reality (SAR). The study conducted with this technology will focus on multimodal analysis in co-design meetings were we have compared two co-design sessions. We notice that the design activity is unchanged by the introduction of such a technology but could encourage interactions from clients who are usually less invested.

Keywords: co-design, spatial augmented reality, design activities, design cognition

\section{Introduction}

The study of design activity emerged in the disciplinary fields of cognitive psychology and ergonomics with the works of Reitman (1964) and Eastman (1969), whose framework was derived from the problem solving approach. The study of these activities addressed very varied fields such as architecture, innovation of industrial products and a more artistic side of the design. Due to the multiplicity of applications of the design and its complexity, researchers rapidly noticed that the classical problem solving approach was not sufficient to understand the design activity in its complexity (especially the creative side), precursors of the 70s have tried to define the notion of "ill defined" design problems (Newell and Simon, 1972; Greeno, 1978; Cross, 1986; Goel and Pirolli, 1992):

- The problems are wide and complex. As a result, the problems are interrelated with many other variables that are difficult to split.

- Therefore, the resolution of these problems requires the pooling of multiple skills requiring the development of collaborations in a group.

- A lot of freedom remains in the start of the design process.

- Multiple solutions to a problem are acceptable: there is not one single "good" solution.

- The steps of problem analysis and problem solving are nested. Thus, the formalized problem does not fully exist prior to the solution, both are interacting.

- The design task is not predefined. It is necessary to combine, reinvent strategies to develop a solution while relying on similar projects and already existing prototypes.

- The evaluation of the solutions is difficult because of the lack of realism of the graphic representations and / or models, and because of criteria that not clearly defined and sometimes 
contradictory. It may be expensive to question the final solutions, which is why we often consider them satisfactory and not optimal.

To overcome the complexity of the design process, traditional design tools such as drawings and sketching have been replaced by technologies to foster the emergence of ideas, support the decisionmaking, help the outsourcing of mental representations and, in our case, for example to improve the involvement of clients in design sessions. Therefore, the objective of our study is to analyse the potential impacts of such a technology on the designer and client's cognitive functions.

As a first step, we will explore the literature in terms of model and method for the analysis of the cognitive process of designers. Then, the section 2 explore the works done around the analysis of gesture in interactions and highlight their importance in the expression of internal representations. We will conclude the state of the art by a brief synthesis of the design assistance systems. The presentation of our methodology will stand in Section 3 before presenting our preliminary results from the observation and analysis of two co-design sessions, one in traditional conditions, the other one using the SAR (Spatial Augmented Reality) technology.

\section{State of the art: The co-design activity}

Collaborative design activities has mainly been studied through the analysis of verbal interactions and gestural interactions.

In our state of the art, we will explore the literature on different models of design activity and then the literature focussing on gestures. We will conclude this part exploring the different existing design assistance tools.

\subsection{Overview of cognitive design activities}

The outcome of empirical - based design research results in cognitive or organisational - models of designing. In order to come up with such proposals for cognitive models, researchers use different empirical methods, the most famous and widely used is protocol analysis. This method is used to characterize design activity by asking designers to solve a design problem where verbal interactions are recorded and transcribed (Ericson and Simon, 1980). Then, according to the coding scheme established for such an experimental case study, the classification results in the extraction of relevant conclusions.

The think aloud method is employed to collect the designers' thoughts during a specific design task. A significant protocol study is reported by Gero and Malher (1997). The aim of this method is to evaluate the time spent on proposing solutions or on reasoning about the function and the behaviour of the design solutions. Think aloud was used to ease the analysis process of the cognitive activities as the designers are asked to verbally express what they are doing. This approach applies well to single design tasks but is not suitable for studying collaborative design.

Lhote et al. (1998), as far as they are concerned, proposed a cognitive design model from the cybernetic point of view. They consider all mental activity as a system where cognitive functions and closely related to another function. The model shows that the iterative behaviour of the designers is combined with the generation of solutions and projection of these solutions to simulate all the possible consequences and compare them to the design specifications (Huysentruyt et al., 2012).

Despite the multitude approaches and methods used by the researchers to study the cognitive design activity, they converge toward the same type of activities:

- the generation activity: elaboration of a new idea, refinement of this idea or proposition of an alternative idea.

- the evaluation activity: evaluation on the basis of predefined criteria.

- The cognitive synchronization: clarification of the subject by the construction of a common reference system.

As considered by Visser (1992), the design task execution activities such as those mentioned above are mixed with management activities: the process-related activities (Stempfke and Badke-Schaub, 2002):

- project management activities

- meeting management activities 
In this paper, we rely on an adapted version of the COMET method. This method is part of the family of protocol analysis methods. It has been designed by researchers from cognitive sciences working in the fiel of design sutides (Darses et al., 2001) and we considered it as a reliable and relevant basis for our analysis framework. The main purpose of COMET is to allow the analysis of the activities implemented by designers during design meetings. This method is based on two steps: 1) dividing speech turns into units, 2) codes these units by type of action (i.e. generation (GEN), evaluation (EVAL), interpretation (INT) and information (INFO)). These actions are associated with the object of the conversation and a distinction is made between the assertion and question.

This method is quite relevant for our case study because it allows to analyze group cognitive design activities in which we are comparing to other method able to relate the cognitive activities only in an individual configuration. We will use this work to create our own coding scheme for verbal interactions between designers and clients but focused on Actions and not on the type of utterance nor the arguments employed.

\subsection{Overview of gestures in design sessions}

Gestural interactions have also been studied in the field of design. Indeed, gestures play a role in communication through the externalizing the stakeholders' thought through the body. The forerunner of gesture studies, McNeill (1992), has suggested that some dimensions of thought are embodied in the gestures. He also identified four categories of gestures that have been taken over and improved in other design researches: iconic, metaphoric, deictic and beat gestures. After him, other gestures' classifications were realized which we could list some of them:

Table 1. Synthesis of gesture classifications

\begin{tabular}{|c|c|c|c|c|c|}
\hline $\begin{array}{c}\text { McNeill } \\
(1992)\end{array}$ & $\begin{array}{c}\text { Bekker et al. } \\
(1995)\end{array}$ & Streeck (2008) & Visser (2009) & $\begin{array}{c}\text { Eris et al. } \\
(2014)\end{array}$ & $\begin{array}{c}\text { Kong et al. } \\
(2015)\end{array}$ \\
\hline Iconic & Kinetic & Modelling & Representation & At sketch & Iconic \\
Metaphoric & Spatial & Bounding & Organization & content & Metaphoric \\
Deictic & Point & Drawing & Focalization & To enact & Deictic \\
Beat & Other & Handling & Modulation & scenario & Emblem \\
& & Making & Disambiguation & At external & Beat \\
& & Scaping & & Nontent & Beat \\
& & Marking & & & identifiable \\
& & Self-marking & & & \\
& & Model-world & & & \\
& & making & & & \\
\hline
\end{tabular}

Several studies on design meetings in the field of architecture and mechanics have analyzed the role of gestures in design. Some studies that focus on individual design (Gero and McNeill, 1998) have described graphical actions. Others, dealing with collaborative design, have analyzed the function of graphic actions and gestures in distant-mediated or face-to-face co-design (Tang, 1991). However, to our knowledge, we have only one study on the intersection of verbal and gestural interactions (Détienne et al., 2006). The latter was using a description language by transcription of the actions. We will use this approach of the interpretation of the verbal exchanges as an inspiration to develop our own gestures' coding scheme.

\subsection{Overview of design assistance systems}

The traditional design tools such as drawing and sketching (Standard) have made way for CAD (Computer Aided Drafting / Design) tools that are used today in every industry. These tools changed the overall design process and the activities of the designers (Darses et al., 2001).

The development of design assistance tools has actually been developed to assist in some of the designer's creative activities. The TRENDS system (Bouchard et al., 2008), for example, is an analog search software that works on the basis of the needs targeted by the users, helping the emergence of creative ideas. 
The T'nD - Touch and Design - design assistance system, for its part, has been designed to externalize ideas and thus improve the transition from an internal representation to a common external representation.

Since decision-making is an important moment in co-design meetings, a software dedicated to this activity has been created (Lockner and Bonnardel, 2014). Using an algorithm, SKIPPI generates ideas and simulates the adequacy of the solution with the expectations of the specifications.

In this context of willingness to assist designers in their activities, Spatial Augmented Reality (SAR) can plays its role, today, in assisting co-design sessions. Indeed, this technology is using projection mapping of a virtual image on a tangible object (Raskar et al., 1998) and makes it possible to apply modifications in real time on the product model and to share an internal representation quasi instantaneously by displaying the modification onto the artefact.

However, to our knowledge, the SAR technology has been used for design purposes only within the SPARK H2020 Project (http://spark-project.net/). The contribution of such technology to the design activity is still to be developed. Therefore, in this study, we will focus on the contributions and impacts of SAR on the multimodal co-design activity, through gesture and speech analysis.

\section{Research question and hypothesis}

Along the integration process of technologies in the design activity, the expert designers have adapted their activities with the use of the digital tools. If they were initially reluctant to use CAD software in the early times, they now use these tools on an everyday basis for modelling products. This has drastically changed the organization of the design process but did it fundamentally changed the cocognitive design activities themselves ? We would like to formulate this question as an hypothesis.

Then, the research question addressed in this paper can be expressed as follows: "Does the use of a Spatial Augmented Reality system modifies the co-design cognitive activity?"

We formulate three hypotheses in connection with our research question:

- H1: The interaction rate of clients and designers is different between SAR condition and standard condition.

- H2: The rate of gestures used by the client and designers are equivalent in the SAR and Standard conditions.

- H3: The rate of co-design cognitive activities of clients and the designers do not significantly vary in the SAR and Standard conditions.

In the next section, we will present the methodology used to generate the required data in order to analyze the whole co-design process from a multimodal point of view.

\section{Methodology}

Two observations of co-design sessions were conducted involving a design agency. One was in standard condition (without specific technology) and the other one was in Spatial Augmented Reality (SAR) condition. We can see an illustration of the design environment configurations on Figure 1.
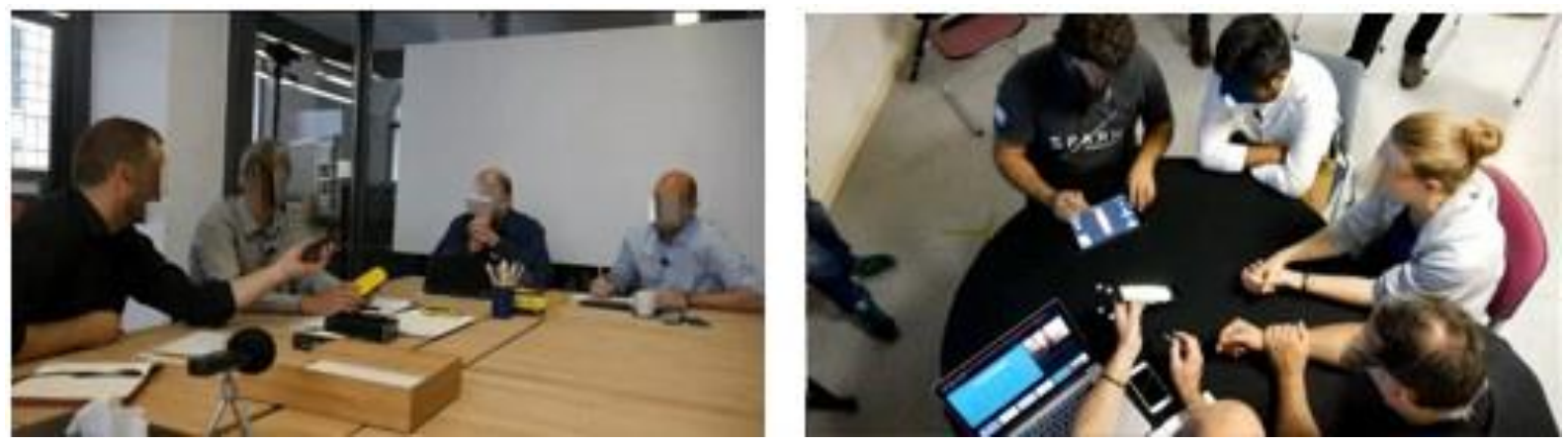

Figure 1. Settings of the Standard (left) and SAR co-design session (right) 


\subsection{Observation framework}

The aim of these two conditions was to collect the characteristics of a SAR session compared to the normal or standard practice of our partner in order to compare the performance of the system with regard to the Standard situation. Therefore, we have created the conditions of the co-design session that are currently carried out at our partners' premises. In these two co-design sessions, two designers worked with their clients in a co-located place where the designers started their meetings by a presentation of design proposals to the clients. Then, the designers collect the client's feedbacks before continuing on a joint discussion on the possible improvements and finishes of the ongoing product. As we are in real industrial environment, the two products are different but we chose two sessions where the products are quite similar in terms of complexity and functionality. In order to be as unobtrusive as possible we use cameras and lapel microphones to save all the necessary data. Each session was completed in 45-90 minutes. Once the codesign sessions are run, we proceeded to a transcription, translation and multimodal analysis based on the co-design cognitive activity model on one hand and the gestures models on the other hand.

\subsection{The co-design cognitive activity}

The co-design cognitive activity metrics were collected thanks to the development of a method inspired by the seminal work of the COMET project presented in section 2 and able to analyse the real-life task-oriented dialogues.

The first phase consists of extracting each individual utterance (turns) every time the locutor changed. While speaking, it's possible that a same actor interact with several things in the scene making gestures through the artefact (tangible, digital, mixed, iconic gestures, metaphoric gestures) or just in the air (gesticulation). Therefore, the utterances are also split every time the object of the interaction change. This turns are as well developed for the analysis of verbal protocol as for gestures. An example of a turns taking split is displayed below:

Table 2. Excerpt of a unit

\begin{tabular}{|l|l|l|}
\hline 00:53:20 & Designer & three LEDs and this other one, \\
\hline 00:53:21 & Designer & which confirms that it is ON ? \\
\hline $00: 53: 22$ & & \\
\hline $00: 53: 23$ & Client & When the light is ... \\
\hline 00:53:26 & & \\
\hline $00: 53: 27$ & Designer & blinking (simulate the blinking) \\
\hline 00:53:28 & Client & blinking/flashing, it means it is activated. The blinking itself does not mean anything. \\
\hline 00:53:29 & Client & It is not six codes, it is three codes; \\
\hline
\end{tabular}

Then, these speech turns are coded according to the type of the unit (if it is an assertion or a request); the cognitive activity corresponding; and the argument which sustain the cognitive activity.

Thus, each turn is coded as TYPE [ACT/ARG] whose the cognitive activity details are listed in the following table:

Table 3. Co-design cognitive activity coding scheme

\begin{tabular}{|c|}
\hline COGNITIVE ACTIVITY \\
\hline Generate [GEN] \\
\hline Inform [INFO] \\
\hline Justify [JUST] \\
\hline Interpret [INT] \\
\hline Evaluate [EVAL] \\
\hline Combine [COM] \\
\hline Simulate [SIM] \\
\hline Acceptance [ACC] \\
\hline Rejection [REJ] \\
\hline Understanding [COMP] \\
\hline
\end{tabular}


The cognitive activity coding scheme established, we proceeded to the validation of this method with a Cohen's Kappa test and Perreault and Leigh inter-coder reliability test. The results of these tests show a substantial agreement (more than $61 \%$ of agreement) between the two different coders in each excerpts (Table 4).

Table 4. Cognitive activity's inter-coder tests

\begin{tabular}{|c|c|c|c|c|}
\hline Session & Coder 1 & Coder 2 & $\begin{array}{c}\text { Perreault et Leigh } \\
(1989) \text { Ir }\end{array}$ & Cohen's Kappa \\
\hline Standard A. & MP & LB & 0,8266 & 0.6546 \\
\hline Standard B. & MP & MT & 0,9493 & 0.7517 \\
\hline SAR & MP & EM & 0,9118 & 0.7220 \\
\hline
\end{tabular}

\subsection{The gestures interactions}

Based on the segmentation of individual utterances, we coded each speech turn thanks to the coding scheme of gestures realized and validated previously. In this study, as we focus on the contents of the interactions and their aim, we are mainly interested in gestures referred to the object. Therefore, the gestures' coding scheme proposed is focusing on gestures made through the artefact but we also take into consideration the gestures in the air. Such a classification is described into seven categories:

- Tangible: Pointing or manipulation towards a model, a prototype, a product or a part of a material product, a sketch, an image, a book, etc. having tangible physical properties in the design environment of designers and clients.

- Digital: Pointing or manipulation of digital equipment such as a television screen, a tablet, a computer, phone and all artefacts displayed on a screen or projected on the wall.

- Mixed: Pointing or manipulation of the physical prototype on which an image is projected. Interaction with a mixed artefact can also be the manipulation of the tablet platform causing changes to the physical prototype.

- Iconic: Such gestures represent images of concrete entities and/or actions. For example, simulating the grasp and bend back something while saying "and he bends it way back." The gesture, as a referential symbol, operates via its formal and structural resemblance to an event or objects.

- Metaphoric: In a metaphoric gesture, an abstract meaning is presented as if it had form and/or occupied space. For example, a speaker appears to be holding an object, as if presenting it, yet the meaning is not presenting an object but an idea or memory of this object.

- Gesticulation: As movements in the air, gesticulations are flicks of the hands up and down or back and forth. This rhythmicity is related to the speech and seems to have a discourse functionality, signalling the temporal locus of something the speaker feels to be important to share.

- None: In this case, no gesture is associated to the speech.

An inter-coder reliability test was also realized in order to validate this gestures coding scheme. The results of the Cohen's Kappa are synthesized in the Table 5 hereafter.

Table 5. Gestures' inter-coder reliability test

\begin{tabular}{|c|c|c|c|c|}
\cline { 2 - 5 } \multicolumn{1}{c|}{} & \multicolumn{2}{c|}{ Standard } & \multicolumn{2}{c|}{ SAR } \\
\hline & Cohen's Kappa & \% agreement & Cohen's Kappa & $\%$ agreement \\
\hline Actor & 0.55 & 73 & 0.71 & 84 \\
\hline Artefact & 0.62 & 74 & 0.59 & 71 \\
\hline
\end{tabular}

Once the two coding schemes were created and validated, the aim was to use these results to analyse and evaluate the impact of a SAR platform on the co-design process and to validate our hypothesis. 


\section{Multimodal analysis and results}

The following section provides an overview of the results obtained for the three different hypothesis.

\subsection{The interaction rate of participants}

Based on the observation of these two co-design situations, we noticed that there is a difference of the interaction rate of clients and designers between the Standard and the SAR session. In the Standard co-design session, the clients interact in $57.4 \%$ of the session time against $75 \%$ in the SAR condition which tends to support the idea of a better performance of SAR for client's participation. Moreover, among the gestures used, we find an equivalence (see Figures 2 and 3) between SAR and Standard conditions for clients or designers except for pointing and / or manipulating of the artefact (tangible, digital, mixed). Indeed, the artefacts being either tangible (Standard condition) or mixed (SAR condition), it seems consistent to find gestures centered on these artefacts according to the condition. SAR technology does not seem to simulate more interaction with the mixed artefact.

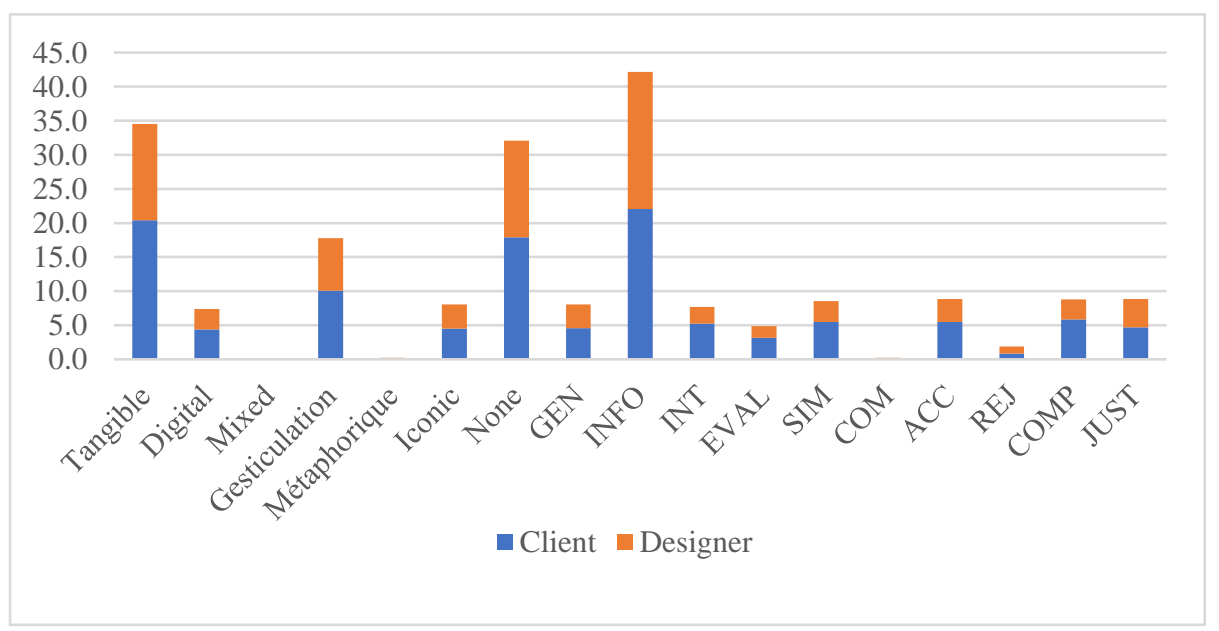

Figure 2. Interaction rate between Clients and Designers in the Standard condition

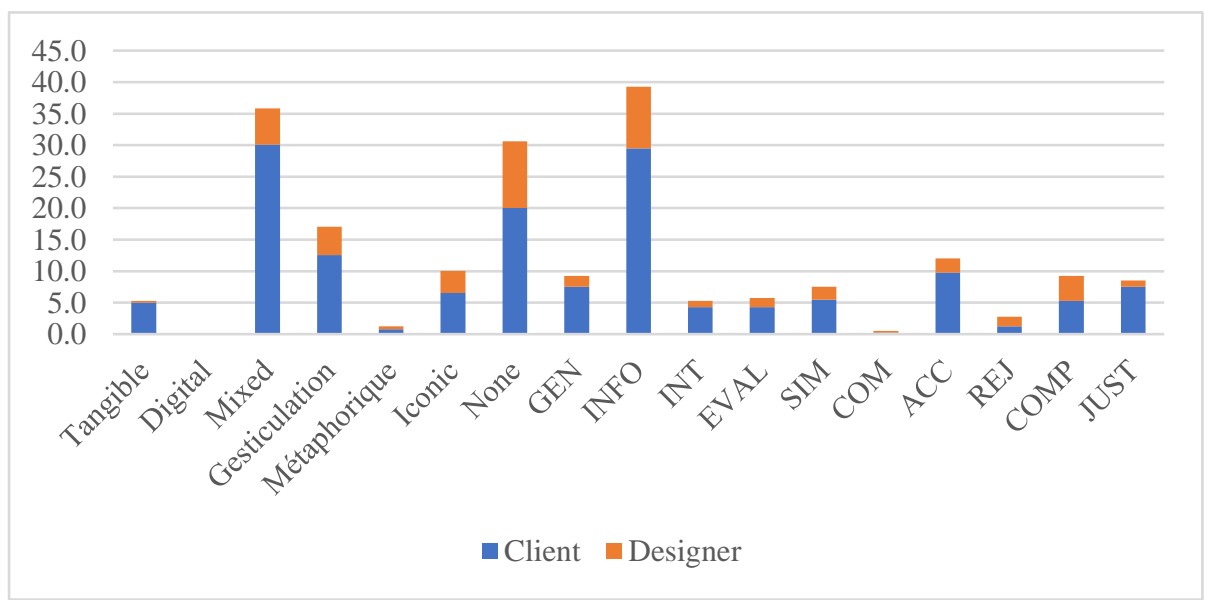

Figure 3. Interaction rate between Clients and Designers in the SAR condition

On the other hand, among the cognitive design activities, we do not notice any difference in cognitive process between a Standard condition and a SAR condition whether for clients or designers except for the case of the provision of information [INFO] for the designers $(9.75 \%$ of interaction in Standard against $20.1 \%$ in the SAR condition). The designers would be more inclined to provide more information about the product in the Standard condition than in the SAR condition. This need to be 
confirmed however by other tests as this effect might be caused by a bias in the observation setting (type of participant, design task, etc.).

It seems that clients interact a little bit more with mixed and tangible objects in SAR session $(\mathrm{Nb} \mathrm{C}$ Tangible 5\%, $\mathrm{Nb} \mathrm{C}$ Mixed 30\%) than in Standard condition (Nb C Tangible 20\%, Nb C Digital 4.5\%) (Figures 2 and 3). Again, this tend to support H1.

We could interpreted these results as a first demonstration of the importance of having a tangible prototype able to be modified in real time externalizing the internal representation of finishing expectations.

\subsection{The gestures' analysis}

Concerning the gestures' analysis according to our two co-design conditions, we have some differences of type of artefact-centred gestures (pointing and/or manipulating of a tangible, digital or mixed artefact) due to the tool available in these sessions. Indeed, the standard condition involves the tangible-basedgestures because the design conditions induce it. In the same way, the SAR condition promoted the use of the mixed artefact as the main external representation of the product, hence mixed gestures predominated. However, the rate of gesticulations, metaphoric gestures, iconic gestures and "None" gestures are quite similar between the Standard and the SAR condition, which tends to support H2.

To resume, the artefact-centered gestures are induced by the external representation available: the "tangible" in a Standard condition and the "mixed" in a SAR condition. Otherwise, we cannot say that the SAR platform has a particular impact on the iconic gestures, metaphoric gestures, gesticulations and "None" gestures made during a co-design session (Table 6).

Table 6. Rate of gestures used between the Standard and the SAR condition

\begin{tabular}{|c|c|c|c|c|c|c|c|}
\hline & Tangible & Digital & Mixed & Gesticulation & Metaphoric & Iconic & None \\
\hline Standard & $34.60 \%$ & $7.30 \%$ & $0 \%$ & $17.80 \%$ & $0.20 \%$ & $8 \%$ & $32.10 \%$ \\
\hline SAR & $5.30 \%$ & $0 \%$ & $35.90 \%$ & $17 \%$ & $1.30 \%$ & $10 \%$ & $30.60 \%$ \\
\hline
\end{tabular}

Whatever the representation used, designers and clients would need gestures to assist the design process.

\subsection{The co-design cognitive analysis}

The rates of the different co-design cognitive activities were listed in the same way as gestures (Table 7). On these results, we observe that the rate of each of the cognitive activities used in the standard condition is equivalent to the SAR condition. For example, we notice that $8.10 \%$ of the standard session is focused on generating an idea [GEN]. This same idea generation activity is occurring in $9.25 \%$ of the SAR session which is quite similar. The most important differences that we can highlight are for the interpretation activities of a concept [INT] and acceptance of an idea [ACC]. However, as these results do not show a notable difference, it is difficult to conclude that the condition of using a SAR tool transform the co-design cognitive activity. This support H3 and tends to confirm that the SAR environment does not impair the cognitive activity of the stakeholders neither it transforms it drastically.

Table 7. Rate of cognitive activities used between the Standard and the SAR condition

\begin{tabular}{|c|c|c|c|c|c|c|c|c|c|c|}
\hline & GEN & INFO & INT & EVAL & SIM & COM & ACC & REJ & COMP & JUST \\
\hline Standard & $8.10 \%$ & $42.20 \%$ & $8.80 \%$ & $5 \%$ & $8.50 \%$ & $0.20 \%$ & $8.90 \%$ & $1.90 \%$ & $8.70 \%$ & $8.90 \%$ \\
\hline SAR & $9.25 \%$ & $39.75 \%$ & $5.25 \%$ & $5.75 \%$ & $7.50 \%$ & $0.50 \%$ & $12.00 \%$ & $2.75 \%$ & $9.25 \%$ & $8.50 \%$ \\
\hline
\end{tabular}

\section{Conclusion}

By introducing the Spatial Augmented Reality technology (SAR) as a tool for co-designing a product, we wondered whether the use of Spatial Augmented Reality had an impact on the design activity of the designers on the one hand and of the clients on the other hand.

First, we were interested in the rate of clients and designers' interactions. Compared to our two codesign sessions, we would tend to say that SAR would increase the customer interaction rate during a 
product design session. We cannot strongly validate our initial hypothesis although the trend is in that direction. Obviously, this is a preliminary result that requires replication to highlight a significant effect of the SAR technology which is not caused by a bias of the design session, actors or products in themselves.

Concerning our second hypothesis on the use of gestures, we can more strongly support this one, which is that each category of gestures is used in equivalent proportions between the Standard and the SAR condition except for pointing gestures and / or manipulation of the artefact. As we saw in section 4 , the predominance of the artefacts depends on the conditions, but the amount remains comparable. This would support the idea that gestures are not dependent of the kind of external representations involved in the co-design sessions.

As for cognitive activities, on the other hand, we show relatively similar proportions of use between Standard and SAR conditions with maximum of differences of 3\% in the information provision activity [INFO], interpretation of a concept [INT] and acceptance of an idea [ACC]. However, these differences in the rate of a cognitive activity are quite small and do not show any particular tendency to be predominantly used in one condition or another. Always with caution for the sake of replicability of results, we can say that there is no significant difference in the rate of cognitive activities between our two conditions of co-design, which supports H3.

In summary, we can conclude that our study does not show any influence of the use of a Spatial Augmented Reality tool in the use of gestures and in the mobilization of cognitive design activities but it could facilitate the commitment of clients through more interactions. This tend to support that the designers' and clients' previous knowledge are strongly internalized in the procedural memory and the tool used would not affect the cognitive process but could facilitate the disinhibition of clients.

We would like to be able to do this same type of analysis on a larger number of co-design sessions in order to bring robustness to our preliminary results.

\section{Acknowledgement}

This paper reported the work from two co-design sessions realized with the Stimulo company. The data we used have been collected during the SPARK project, which received funding from the European Union's Horizon 2020 research and innovation program under grand agreement No. 688417 . We really would like to thanks them for the availability of the designers and the richness of the work done which allowed us to be closer to the applied field. We also would like to thank all those who, in one way or another, contributed to the development of the coding scheme and the collection of data.

\section{References}

Bekker, M.M., Olson, J.S. and Olson, G.M. (1995), “Analysis of gestures in face-to-face design teams provides guidance for how to use groupware in design”, In DIS '95 Proceedings of the 1st conference on Designing interactive systems: processes, practices, methods, \& techniques, ACM, New York, US, pp. 157-166.

Bouchard, C. et al. (2008), "TRENDS: A content-based information retrieval system for designers", Proceedins of the 3rd International Conference on Design Computing and Cognition, Atlanta, USA.

Cross, N. (1986), "Understanding design: the lessons of design methodology", Design Methods and Theories, Vol. 20 No. 2, pp. 409-438.

Darses, F. et al. (2001), "COMET: A method for analysing collective design processes", Rapport de recherché $n^{\circ} 4258$, INRIA, Rocquencourt, France. Also accessible at http://hal.inria.fr/inria-00072330/en/

Détienne, F., Visser, W. and Tabary, R. (2006), "Articulation des dimensions graphico-gestuelle et verbale dans l'analyse de la conception collaborative", Psychologie de l'interaction, L'Harmattan, 2006, Langage et cognition : Contraintes pragmatiques, pp. 283-307.

Eastman, C.M. (1969), "Cognitive processes and ill-defined problems: a case study from design", In Proceedings of IJCAI 69, pp. 669-690.

Ericsson, K.E. and Simon, H.A. (1980), “Verbal reports as data”, Psychological Review, Vol. 873, pp. $215-251$.

Eris, O., Martelaro, N. and Badke-Schaub, P. (2014), A comparative analysis of multimodal communication during Design Sketching in co-located and distributed environments, Design Studies, In Press, pp. 1-34. https://doi.org/10.1016/j.destud.2014.04.002

Gero, J.S. and Maher, M.L. (1997), A framework for research in design computing, in Martens B., Linzer H. and Voigt A. eds, ECCADE'97. 
Gero, J.S. and McNeill, T. (1998), “An approach to the analysis of design protocols”, Design Studies, Vol. 19 No. 1, pp. 21-61.

Goel, V. and Pirolli, P. (1992), “The structure of Design Problem Spaces”, Cognitive Science, Vol. 16, pp. 395-429.

Greeno, J.G. (1978), "Natures of problem-solving abilities", Handbook of Learning and Cognitive Processes, Vol. 5, Lawrence Erlbaum, Hillsdale, N.J., pp. 239-270.

Huysentruyt, J., Lespinet-Najib, V. and Chen, D. (2012), "A model of cognitive activities in design", 9th International Conference on Modeling, Optimization, Bordeaux, France.

Kong, A.P.-H. et al. (2015), "A coding system with independent annotations of gesture forms and functions during verbal communication: Development of a database of speech and gesture (DoSaGE)", Journal of nonverbal behavior, Vol. 39 No. 1, pp. 93-111.

Lhote, F., Chazelet, Ph., and Dulmet, M. (1998). "Elargissement de principes de la cybernétique vers l'ingénierie de la production", INCOM'98, Nancy, June 23-25, 1998.

Lockner, D. and Bonnardel, N. (2014). "Emotion and Interface Design: How web design may elicit positive user affect?", In proceedings of the 5th International Conference on KEER'2014, Kaisei Engineering \& Emotion Research, Linköping University, Sweden (2014).

McNeill, D. (1992), Hand and Mind: What gestures reveal about thought, University of Chicago Press, Chicago.

Newell, A. and Simon, H. A. (1972), Human problem solving. Prentice-Hall, Englewood Cliffs, N.J.

Reitman, W. (1964), "Heuristic decision procedures, open constraints, and the structure of ill-defined problems", In: Shelley, M.W. and Bryan, G.L. (Eds), Human judgment and optimality, Wiley, New York.

Raskar, R., Welch, G. and Fuchs, H. (1998), "Spatially augmented reality", In: Proceeding of the First IEEE Workshop on Augmented Reality, Vol. 1998 No. November 1, San Francisco, CA, pp. 63-72.

Streeck, J. (2008). "Depicting by gesture", Gesture, Vol. 8 No. 3, pp. 285-301. https://doi.org/10.1075/ gest.8.3.02str

Stempfle, J. and Badke-Schaub, P. (2002), "Thinking in design teams - an analysis of team communication", Design studies, Vol. 23, pp. 473-496.

Tang, J. (1991), "Findings from observational studies of collaborative work", International Journal of ManMachine Studies, Vol. 34 No. 2, pp. 143-160. Retrieved from http://www.sciencedirect.com/science/ article/pii/002073739190039A

Visser, W. (1992), "Design Organisation: There is more to expert knowledge than is dreamed of in the planner philosophy", Rapport de Recherche $n^{\circ} 1765$, INRIA, October 1992.

Visser, W. (2009), "Form and function of gestures in an architectural design meeting", In: McDonnell, J. and Lloyd, P. (Eds.), About Designing: Analysing Design Meetings, Taylor \& Francis, London, UK, pp. 269-284. 